\title{
British Undergraduate Dental Research Conference 2020
}

\section{By Christina Wainer, 5th year BDS student, University of Bristol}

Building on the success of their previous conferences, the Manchester Undergraduate Dental Research Society (MUDRSoc) organised the fifth annual British Undergraduate Dental Research Conference (BUDRC). The event took place on 29 February 2020 at Manchester's University Place. The 2020 conference welcomed more than 300 delegates from 15 dental schools across the UK.

The day began with 'DentTalks', which are short lectures delivered in a TED-talk style. The talks covered the latest advancements in research, including 'Caries Diagnosis: Don't believe everything you are told - look at the evidence,' 'Oral cancer: Establishing a global network of innovators' and 'Stem cells in dentistry: How close are we?'. These informative DentTalks were delivered by Professor David Ricketts, Dr Niall Mc Goldrick, and Dr Evgeny Kushnerev, respectively.
A new element to the conference this year was the introduction of a poster display. Selected posters encompassed a range of dental topics and could be either a clinical case report, an audit, or a research project. The posters were on display throughout the day, with authors undertaking their poster presentation on their chosen topic in the early afternoon.

The remainder of the afternoon consisted of numerous workshops, covering an array of disciplines led by skilled clinicians and experts in their respective fields. Many offered hands-on learning opportunities, including anterior composite build-ups, suturing, dental photography, implants, digital impression taking and splinting. Occlusion, management of tooth surface loss and power of social media in dentistry were just some of the other options on offer.

The conference's DentTalks and poster presentations highlight the latest advancements in dentistry, whilst the

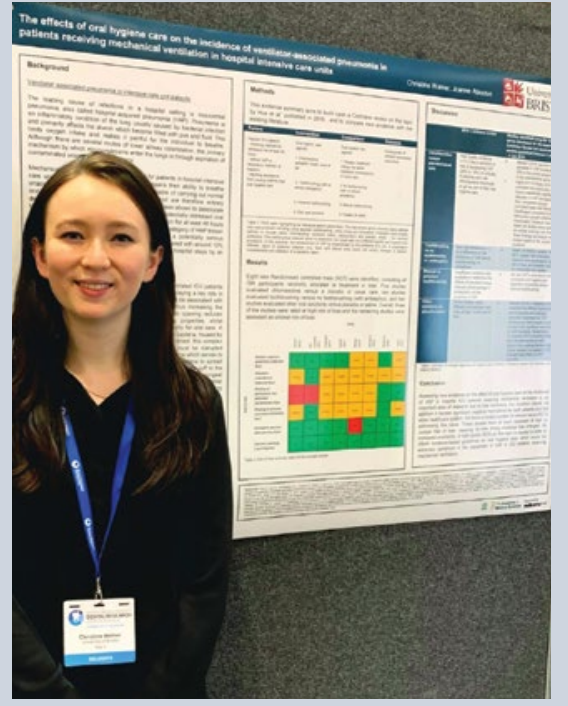

afternoon workshops permit attendees to further develop core practical skills. MUDRSoc has provided a fantastic opportunity for sixth formers, undergraduate students, foundation year dentists and postgraduates to explore what a career in academia would entail.

\section{A lifesaving update for the dental team}

Sarah McKernon, a Clinical Lecturer/

Specialist in Oral Surgery, and Mustafa Thwaini, a BDS student, from the University of Liverpool, have written to the $B D J$ to update the readership about recent changes to out of hospital management of cardiac arrest during the COVID-19 pandemic, as recommended by the Resuscitation Council UK.

This is essential information for the dental team.

RCUK guidelines in summary:

1. When recognising a cardiac arrest, do not listen or feel for breathing by placing your ear or cheek close to the patient's mouth. If in doubt about confirming cardiac arrest, then start chest compressions until help arrives - only begin to do this if there is no perceived risk of COVID-19 infection. If there is a perceived risk of COVID-19 infection, place a cloth over the patient's mouth and nose prior to starting chest compressions

2. As always, make sure an ambulance is on its way. If the patient is suspected or known COVID-19 positive, then inform 999 when you call for help

3. Use a defibrillator if you have access to one. Early defibrillation significantly improves chances of a positive outcome for both the person in cardiac arrest and the healthcare professional (HCP) with regards to COVID-19 infection

4. If you have access to personal protective equipment (PPE) (ie FFP3 face mask, gloves, eye protection) wear it throughout

5. After performing 'compression only CPR', it is important that the team thoroughly wash their hands and arms with soap and water. They should also seek advice from the NHS 111 if COVID-19 infection is suspected.

The Resuscitation Council UK have produced a helpful video that can be found on their website: https://bit.ly/35TITYd. ${ }^{1}$

It is important to note that until recently both Resuscitation Council UK and Public Health England (PHE) have had differing opinions on the level of PPE required for chest compressions. This has caused a lot of confusion across the NHS posing the question 'which guidelines are the correct guidelines to follow?'. A recent statement on 24 April aligns PHE guidelines with those of RCUK, ${ }^{2}$ allowing Trusts to adopt AGP PPE if they consider it more appropriate. RCUK urge HCPs to relay this information to their trusts to best ensure their safety, as well as that of their colleagues and families.

With thanks to Miss McKernon and Mr Thwaini for this update.

\section{References}

1. Resuscitation Council (UK). Resuscitation Council UK Statement on COVID-19 in relation to CPR and resuscitation in first aid and community settings. 22 March 2020. Available at: https://www.resus.org.uk/media/ statements/resuscitation-council-uk-statements-on-covid-19-coronavirus-cpr-and-resuscitation/covid-community/ (accessed May 2020)

2. Resuscitation Council (UK). Statement on PHE PPE Guidance. 24 April 2020. Available at: https://www. resus.org.uk/media/statements/resuscitation-council-uk-statements-on-covid-19-coronavirus-cpr-and-resuscitation/statement-on-phe-ppe-guidance (accessed 28 April 2020). 